\title{
Elastase activity on sputum neutrophils correlates with severity of lung disease in cystic fibrosis
}

\author{
A. Susanne Dittrich ${ }^{1,2,3,4}$, Iris Kühbandner ${ }^{1,2,3}$, Stefanie Gehrig ${ }^{1,2,4,5}$, \\ Verena Rickert-Zacharias ${ }^{1,2,4,5}$, Matthew Twigg ${ }^{6}$, Sabine Wege ${ }^{2,3}$, \\ Clifford C. Taggart ${ }^{6}$, Felix Herth ${ }^{2,3}$, Carsten Schultz ${ }^{2,4,5}$ and \\ Marcus A. Mall $1,2,4,7,8$
}

Affiliations: ${ }^{1}$ Dept of Translational Pulmonology and Division of Paediatric Pulmonology and Allergy and Cystic Fibrosis Centre, University of Heidelberg, Heidelberg, Germany. ${ }^{2}$ Translational Lung Research Centre Heidelberg (TLRC), German Centre for Lung Research (DZL). Heidelberg, Germany. ${ }^{3}$ Dept of Pneumology and Critical Care Medicine, Thoraxklinik at the University Hospital Heidelberg, Heidelberg, Germany. ${ }^{4}$ Molecular Medicine Partnership Unit (MMPU). University of Heidelberg and European Molecular Biology Laboratory, Heidelberg, Germany. ${ }^{5}$ Cell Biology and Biophysics Unit, European Molecular Biology Laboratory, Heidelberg, Germany. 'Airway Innate Immunity Group (AilR), Centre for Experimental Medicine, Queen's University Belfast, Belfast, UK. ${ }^{7}$ Dept of Paediatric Pulmonology and Immunology, Charité - Universitätsmedizin Berlin, Berlin, Germany. ${ }^{8}$ Berlin Institute of Health (BIH), Berlin, Germany.

Correspondence: Marcus A. Mall, Dept of Paediatric Pulmonology and Immunology, Charité Universitätsmedizin Berlin, Augustenburger Platz 1, 13353 Berlin, Germany. E-mail: Marcus.mallacharite.de

@ERSpublications

Surface-bound elastase activity on sputum neutrophils correlates with severity of lung disease in cystic fibrosis http://ow.ly/SRcT30iziHe

Cite this article as: Dittrich AS, Kühbandner I, Gehrig S, et al. Elastase activity on sputum neutrophils correlates with severity of lung disease in cystic fibrosis. Eur Respir J 2018; 51: 1701910 [https://doi.org/ 10.1183/13993003.01910-2017].

ABSTRACT Neutrophil elastase (NE) is a key risk factor for severity of cystic fibrosis (CF) lung disease. Recent studies identified increased NE activity on the surface of airway neutrophils from CF-like mice and patients with CF. However, the role of surface-bound NE in CF lung disease remains unknown. We determined the relationship between surface-bound NE activity and severity of lung disease in CF.

Surface-bound NE activity was measured on sputum neutrophils from 35 CF patients and eight healthy controls using novel lipidated Förster resonance energy transfer reporters and correlated with free NE activity, neutrophil counts, interleukin-8, myeloperoxidase and antiproteases in sputum supernatant, and with lung function parameters.

Surface-bound NE activity was increased in CF compared to healthy controls $(\mathrm{p}<0.01)$ and correlated with free NE activity $(p<0.05)$ and other inflammation markers $(p<0.001)$. Surface-bound and free NE activity correlated with forced expiratory volume in $1 \mathrm{~s} \%$ predicted $(\mathrm{p}<0.01$ and $\mathrm{p}<0.05)$, but only surfacebound NE activity correlated with plethysmographic functional residual capacity \% pred $(\mathrm{p}<0.01)$ in patients with CF.

We demonstrate that surface-bound NE activity on airway neutrophils correlates with severity of lung disease in patients with CF. Our results suggest that surface-bound NE activity may play an important role in the pathogenesis and serve as novel biomarker in CF lung disease. 


\section{Introduction}

Increased activity of neutrophil elastase (NE), a major product of activated neutrophils, has been identified as a key risk factor for the onset and progression of bronchiectasis and lung function decline in patients with cystic fibrosis (CF) and non-CF bronchiectasis [1-5]. Beyond structural damage of airway walls, NE has been implicated in the pathogenesis of mucus hypersecretion [6, 7], airway inflammation [8-11] and impaired defences against Pseudomonas aeruginosa infection [11-14]. In addition, increased $\mathrm{NE}$ activity was shown to disable $\mathrm{CF}$ transmembrane conductance regulator chloride channels and activate epithelial sodium channels $(\mathrm{ENaC})$, thus aggravating basic defects in anion secretion and sodium ion absorption in CF airways [15-17]. These findings suggest NE activity in sputum or bronchoalveolar lavage (BAL) fluid as a promising biomarker of neutrophilic inflammation in CF lung disease [2-4]. So far, measurements have focused on the detection of free $\mathrm{NE}$ activity in BAL fluid and sputum supernatant $[2-4,18]$. Since NE is a highly cationic molecule, a significant proportion of secreted $\mathrm{NE}$ is bound to the neutrophil membrane via electrostatic interactions $[19,20]$. Using a novel Förster resonance energy transfer (FRET)-based NE reporter assay [21], we demonstrated that NE activity is increased on the surface of airway neutrophils in patients with CF [22]. In addition, we showed that elevated surface-bound NE activity is implicated in airway inflammation, mucus hypersecretion and structural lung damage in mice with CF-like lung disease, even in the context of moderate airway neutrophilia, where released NE is effectively inhibited by an intact antiprotease shield and no free NE activity is detectable in BAL fluid [22]. However, the role of $\mathrm{NE}$ activity on the neutrophil surface in CF lung disease remains unknown. Based on these previous findings in mice with CF-like lung disease [22], we hypothesised that surface-bound NE activity is associated with severity of lung disease in patients with CF. To test this hypothesis, we collected sputum from a cohort of 35 patients with CF and eight healthy nonsmokers and used FRET reporters to quantify surface-bound NE activity on sputum neutrophils and free NE activity in sputum supernatants. To determine the relationship of these different pools of NE activity with airway inflammation and proteolytic burden, we correlated surface-bound and free NE activity with sputum neutrophil counts, levels of interleukin (IL)-8, myeloperoxidase (MPO) and antiproteases such as $\alpha_{1}$-antitrypsin-NE complexes (AAT-NE) and secretory leukocyte protease inhibitor (SLPI) in sputum supernatant. Furthermore, we performed paired spirometry and body plethysmography and correlated surface-bound and free NE activity with forced expiratory volume in $1 \mathrm{~s}$ (FEV1) as a global parameter of lung disease severity and plethysmographic functional residual capacity (FRCpleth) as a parameter of air trapping in patients with CF.

\section{Methods}

\section{Study population}

This observational study was approved by the ethics committee of the University of Heidelberg (Heidelberg, Germany) and informed written consent was obtained from all subjects. The diagnosis of CF was verified by established diagnostic criteria [23]. Spontaneously expectorated $(n=36)$ or induced $(n=3)$ sputum of sufficient quantity ( $>60000$ total inflammatory cells) was collected from 35 clinically stable patients with CF during routine visits to the CF centre at the University Hospital Heidelberg (table 1 and online supplementary table S1). Colonisation with $P$. aeruginosa was defined as negative, intermittent and chronic when $0 \%, \leqslant 50 \%$ and $>50 \%$ of airway cultures, respectively, were positive in the previous

Support statement: Funded in part by the German Ministry for Education and Research (FKZ 82DZL00401 and FKZ 82DZL004A1 to M.A. Mall and C. Schultz), the European Commission (Seventh Framework Programme Project No. 603038 CFMatters to M.A. Mall) and the German Cystic Fibrosis Association Mukoviszidose e.V. (Project number 1605 to A.S. Dittrich). A.S. Dittrich was supported by a HRCMM (Heidelberg Research Centre for Molecular Medicine) career development fellowship. Funding information for this article has been deposited with the Crossref Funder Registry.

Conflict of interest: A.S. Dittrich reports grants from the German Cystic Fibrosis Association Mukoviszidose e.V (number 1605) and the Heidelberg Research Centre for Molecular Medicine Career Development Fellowship, during the conduct of the study. F. Herth has received grants, personal fees and non-financial support from Novartis, Chiesi, Boehringer, Astra, Uptake, Broncus, Pulmonx, Olympus, BTG and Ethicon, outside the submitted work. C. Schultz co-founded SiChem in 2001 and still own part of the company. SiChem is the sole producer of FRET probes used in this manuscript. M.A. Mall received grants from the German Federal Ministry of Education and Research (grant contract number 82DZL00401 and 82DZL004A1) and the European Commission (Seventh Framework Programme Project number 603038 CFMatters), during the conduct of the study; and personal fees for advisory board participation, consultancy, and lecture fees from Vertex, personal fees for advisory board participation and consultancy, from Spyrex, Polyphor and Boehringer Ingelheim, personal fees for advisory board participation from ProQR, PTC Pharmaceuticals, Arrowhead and Pro Axis, and lecture fees from Bayer, outside the submitted work. In addition, M.A. Mall has a patent on the Scnn1b-transgenic mouse with royalties paid, and a patent on use of sodium channel blockers for early therapy of obstructive lung diseases issued. 


\section{TABLE 1 Clinical characteristics of patients with cystic fibrosis}

\begin{tabular}{|c|c|}
\hline Subjects & 35 \\
\hline Visits & 39 \\
\hline \multicolumn{2}{|l|}{ Age years } \\
\hline Median (IQR) & $27.7(23.8-30.8)$ \\
\hline Range & $19.1-59.0$ \\
\hline Male/female & $23 / 12$ \\
\hline \multicolumn{2}{|l|}{ BMI kg·m ${ }^{-2}$} \\
\hline Median (IQR) & $19.8(18.9-21.4)$ \\
\hline Range & $16.3-27.7$ \\
\hline \multicolumn{2}{|l|}{ FEV $1 \%$ pred } \\
\hline Median (IQR) & $53.3(38.7-68.1)$ \\
\hline Range & $18.0-114.8$ \\
\hline \multicolumn{2}{|l|}{ FRCpleth \% pred ${ }^{\#}$} \\
\hline Median (IQR) & $140.4(113.8-169.4)$ \\
\hline Range & $89.6-212.4$ \\
\hline \multicolumn{2}{|l|}{ CFTR genotype } \\
\hline F508del/F508del & $13 / 35(37.1)$ \\
\hline F508del/other & 19/35 (54.3) \\
\hline Other/other & $3 / 35(8.6)$ \\
\hline \multicolumn{2}{|l|}{ Pseudomonas infection } \\
\hline Negative & 13/39 (33.3) \\
\hline Intermittent & $3 / 39(7.7)$ \\
\hline Chronic & 23/39 (59.0) \\
\hline Pancreatic insufficiency & $30 / 35$ (83.3) \\
\hline
\end{tabular}

Data are presented as $\mathrm{n}$ or $\mathrm{n}(\%)$, unless otherwise stated. IQR: interquartile range; BMI: body mass index; FEV1: forced expiratory volume in $1 \mathrm{~s}$; FRCpleth: plethysmographic functional residual capacity; CFTR: cystic fibrosis transmembrane conductance regulator. " : available in 28 of 39 visits.

12 months. As a control group, we collected induced sputum from eight age-matched, healthy, nonsmoking volunteers (five females and three males; median (range) age 31.1 (22.0-45.0) years) by hypertonic saline inhalation as previously described $[22,24]$. In patients with CF, we performed spirometry to determine $\mathrm{FEV} 1$ as a global parameter of lung function and body plethysmography to determine FRCpleth as a parameter of air trapping on the same day of sputum collection, according to American Thoracic Society/European Respiratory Society guidelines [25, 26].

\section{Sputum processing and measurements of free and surface-bound NE activity, cytokines and} antiproteases

Sputum was separated from saliva and homogenised using 10\% sputolysin (Calbiochem, Darmstadt, Germany). Sputum inflammatory cells were isolated and total immune cell counts were performed. Free NE activity was quantified in sputum supernatants using the FRET probe NEmo-1 and compared with activity levels determined by the chromogenic substrate MeO-Suc-AAPV-pNA (Sigma, St Louis, MO, USA) [1-3]. Surface-bound NE activity on sputum neutrophils was measured using the lipidated FRET reporter NEmo-2 [21, 22] and determined from the ratio of donor to acceptor fluorescence as previously described and detailed in the online supplementary material[22]. Levels of IL-8, MPO, SLPI and AAT-NE were measured using ELISA. Differential cell counts were performed on May-Grünwald-Giemsa-stained cytospin preparations. For three CF and one control subject, differential cell count could not be determined because the cytospins were of poor quality. Values below the detection limit are reported as 0 . Additional information is provided in the online supplementary material.

\section{Statistical analysis}

Data were analysed using R ( $\mathrm{R}$ Foundation for Statistical Computing, Vienna, Austria) or SigmaPlot (Systat Software, Erkrath, Germany) and are reported as median (25-75th percentile). Statistical analysis was performed using Shapiro-Wilk tests, Wilcoxon rank sum tests, one-way ANOVA and Kruskal-Wallis tests. Correlation analyses were performed using the Spearman rank order method. A p-value $<0.05$ was considered statistically significant. In the case of multiple comparisons, individual p-levels are indicated only if not rejected by the Bonferroni-Holm method. 


\section{Results}

NE activity is increased on the surface of sputum neutrophils in CF, even in patients with low levels of free NE activity in sputum supernatant

To determine surface-bound NE activity on airway neutrophils and its relationship with free NE activity in CF airway inflammation, we assessed sputum samples from 35 clinically stable adult patients with CF (table 1) and eight healthy nonsmokers using highly sensitive NE-specific FRET reporters developed to quantify free (NEmo-1) and surface-bound (NEmo-2) NE activity [21, 22]. As expected, absolute neutrophil counts $(\mathrm{p}<0.001)$ and percentage of neutrophils $(\mathrm{p}<0.001)$ in sputum were significantly increased in our cohort of adult patients with CF compared to controls (figure 1a and b). Free NE activity detected by NEmo-1 was significantly increased in CF compared to control sputum $(\mathrm{p}<0.01)$, completely inhibited by the NE inhibitor sivelestat (figure 1d) and showed a strong correlation with free NE activity detected by the established chromogenic substrate MeO-Suc-AAPV-pNA $(r=0.91, p<0.001$; online supplementary figure S1). Similar to free NE activity, surface-bound NE activity was significantly increased on CF compared to control neutrophils $(\mathrm{p}<0.01)$ and completely inhibited by sivelestat (figures $1 \mathrm{c}$ and 1e). Surface-bound and free NE activity showed a moderate correlation in CF samples ( $p<0.05$; figure 1f). Of note, grouping of CF samples according to the levels (quartiles) of free NE activity in sputum supernatant demonstrated that surface-bound NE activity was significantly increased on CF compared to control neutrophils, even in patients with low levels ( $\leqslant 25$ th percentile) of free NE activity $(\mathrm{p}<0.01$; figure $1 \mathrm{~g}$ ). Taken together, these results demonstrate that, in addition to free NE activity in airway secretions, surface-bound NE activity on airway neutrophils is increased and contributes to the protease burden in the lungs of patients with CF.

\section{Relationship of surface-bound and free NE activity with markers of neutrophilic inflammation and antiproteases}

Next, we determined the relationship of free and surface-bound NE activity in sputum with markers of neutrophilic inflammation including neutrophil counts, IL-8 and MPO, and antiproteases that inhibit NE activity in the airway lumen. Free NE activity in sputum supernatants correlated with absolute neutrophil counts, IL-8 and MPO across the entire study population, as well as in the CF group only (figure $2 \mathrm{a}, \mathrm{c}$ and e). Surface-bound NE activity correlated with these markers of neutrophilic inflammation across the entire study population (figure $2 \mathrm{~b}, \mathrm{~d}$ and $\mathrm{f}$ ). In the $\mathrm{CF}$ group, higher levels of surface-bound $\mathrm{NE}$ activity tended to correlate with higher levels of neutrophils, IL-8 and MPO; however, these weaker associations did not reach statistical significance for the number of patients included in this study (figure $2 \mathrm{~b}, \mathrm{~d}$ and $\mathrm{f}$ ). Neither free nor surface-bound NE activity correlated with percentage of sputum neutrophils in patients with $\mathrm{CF}$ (online supplementary figure S2). To investigate the relationship of free and surface-bound NE activity with antiproteases, we measured levels of SLPI and AAT-NE, two major endogenous inhibitors of NE in the airways [27], in sputum supernatants. As expected [12, 28], AAT-NE was significantly increased $(\mathrm{p}<0.001)$, whereas SLPI was decreased $(\mathrm{p}<0.001)$ in CF compared to control sputum (table 2). Free $\mathrm{NE}$ activity correlated with AAT-NE across the entire study population, as well as the CF group only (figure 3a). In addition, surface-bound NE activity correlated with AAT-NE across the entire study population and showed a weak association in the CF group (figure $3 \mathrm{~b}$ ). Both free and surface-bound NE activity correlated inversely with SLPI across the entire study population and showed a similar trend that did not reach statistical significance in the CF group (figure $3 c$ and $d$ ).

\section{Surface-bound NE activity on sputum neutrophils correlates with lung function and air trapping}

To determine the clinical relevance of increased NE activity on the surface of CF neutrophils, we correlated free and surface-bound NE activity with FEV1 as a global parameter of lung function and FRCpleth as a parameter of air trapping in patients with CF. As expected [3, 4], free NE activity in CF sputum showed a negative correlation with $\mathrm{FEV}_{1} \%$ pred $(\mathrm{p}<0.05)$ in our cohort of patients with $\mathrm{CF}$ (figure 4a), whereas no relationship was found between free $\mathrm{NE}$ and FRCpleth \% pred (figure 4c). Surface-bound NE activity on CF sputum neutrophils was inversely correlated with FEV1 \% pred ( $\mathrm{p}<0.01$; figure $4 \mathrm{~b}$ ). In addition, surface-bound NE activity was directly correlated with FRCpleth $\%$ pred in patients with $\mathrm{CF}(\mathrm{p}<0.01$; figure $4 \mathrm{~d})$.

\section{Discussion}

This is the first study to examine the role of membrane-bound NE activity on the surface of airway neutrophils in CF lung disease. Chronic neutrophilic airway inflammation in CF is associated with increased levels of free NE activity in the extracellular compartment of the airway lumen, as well as increased expression of membrane-bound NE on the surface of activated airway neutrophils [2, 3, 22, 29, 30]. Despite detection of NE activity in these two distinct compartments, previous studies examining the role of NE in CF lung disease were limited to measurements of free NE activity in airway secretions and 

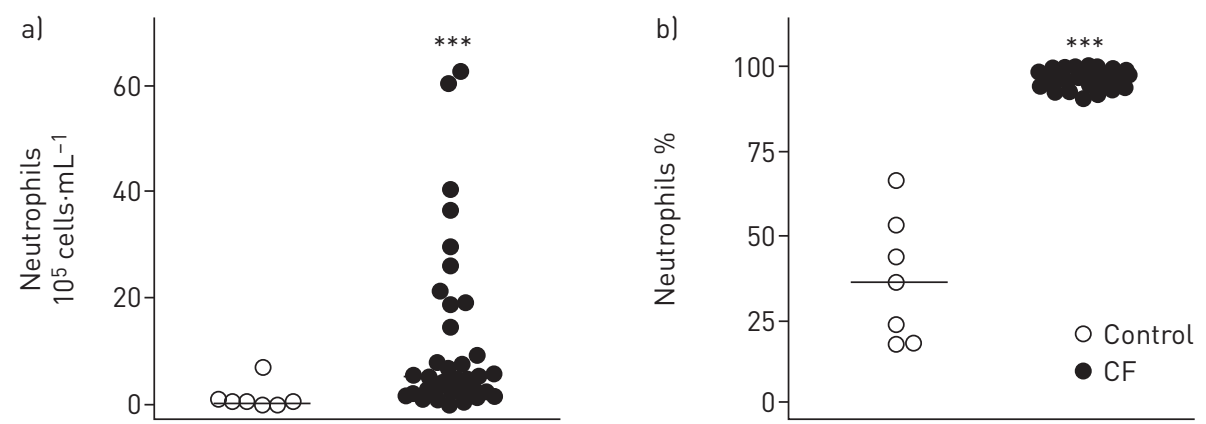

c)
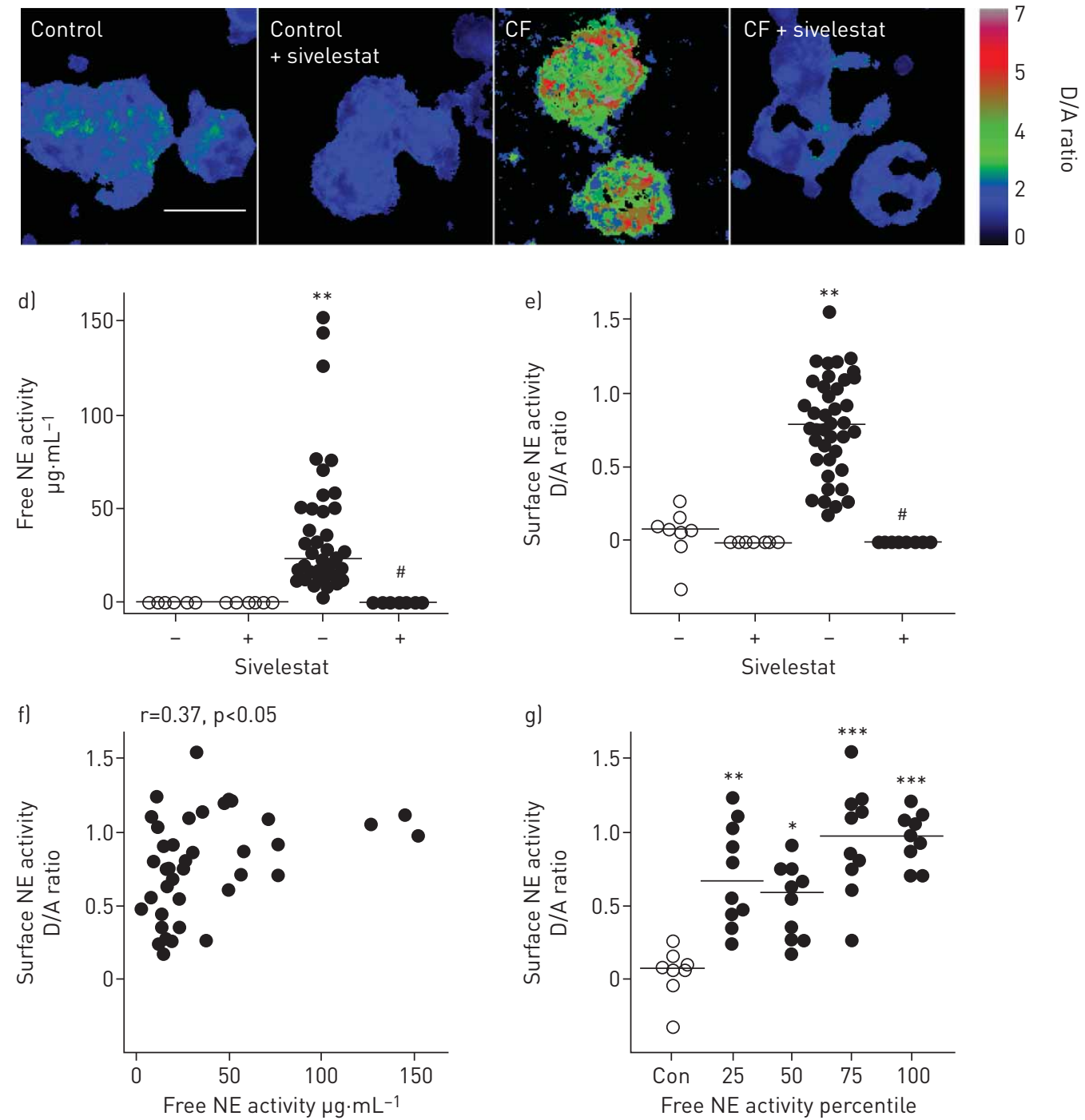

FIGURE 1 Neutrophil elastase (NE) activity is increased on the surface of cystic fibrosis (CF) sputum neutrophils. a) Absolute and b) relative numbers of neutrophils in control and CF sputum. c) Representative ratio images calculated from donor and acceptor fluorescence of sputum neutrophils from a healthy nonsmoker (control) and a patient with CF. Scale bar $=15 \mu \mathrm{m}$. d) Free NE activity in sputum supernatants and e) cell surface-bound NE activity on sputum neutrophils from controls and patients with CF in the absence and presence of the NE inhibitor sivelestat. f) Correlation between surface-bound and free NE activity in CF sputum. g) Surface-bound NE activity on sputum neutrophils from controls (Con) and from patients with CF stratified according to quartile groups of free NE activity in sputum supernatant (25: $\leqslant 25$ th percentile; 50 : $>25-50$ th percentile; 75: $>50-75$ th percentile; 100: $>75-100$ th percentile). D/A: donor/acceptor fluorescence. Data are presented as individual samples and group medians. ${ }^{*}: p<0.05 ;{ }^{* *}: p<0.01 ;{ }^{* * *}: p<0.001$ compared with control; ${ }^{\#}$ : $p<0.001$ compared with CF without sivelestat. 

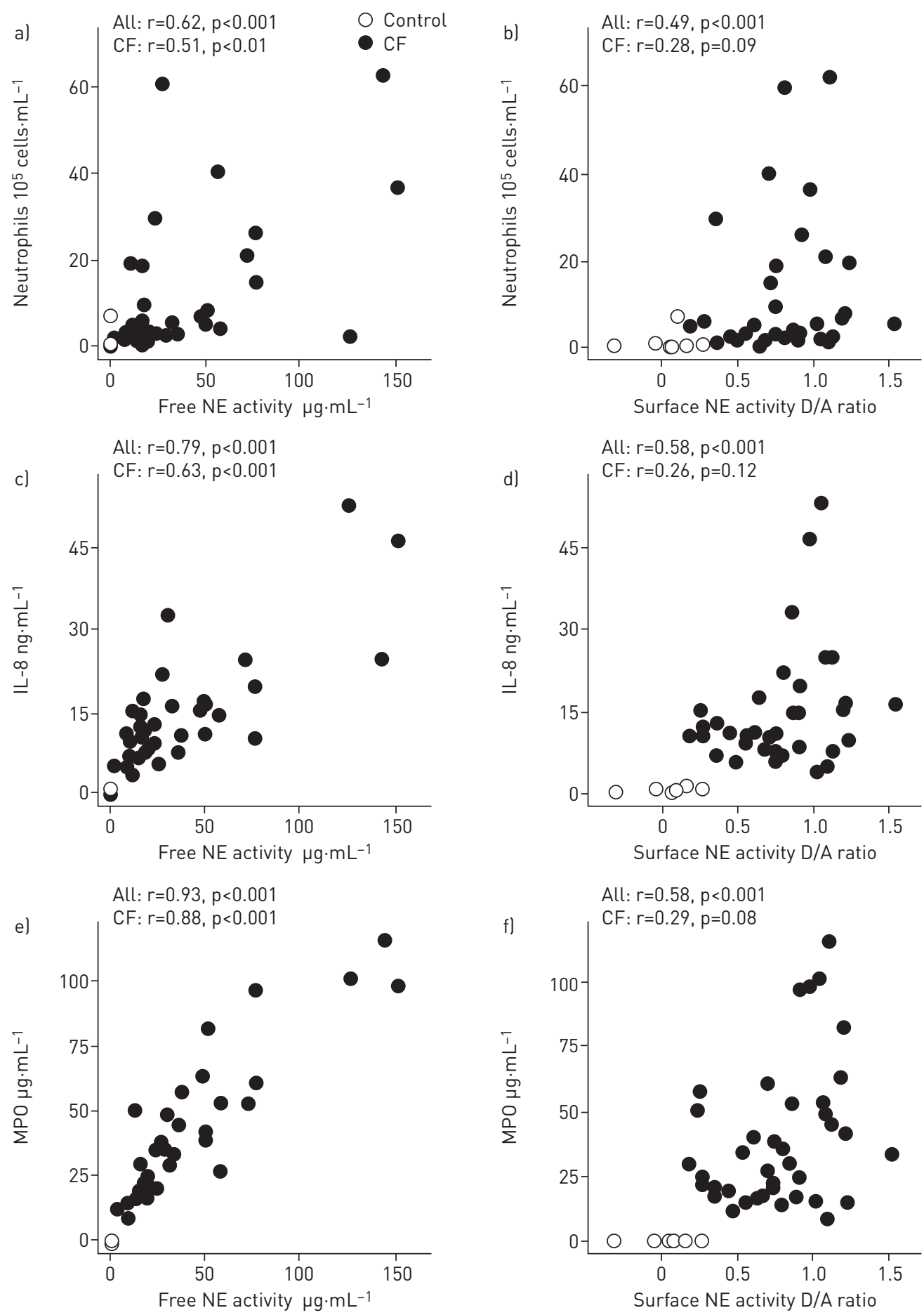

FIGURE 2 Relationship of free and surface-bound neutrophil elastase (NE) activity with parameters of neutrophilic inflammation in sputum from the entire study population (all: control and cystic fibrosis (CF)) and patients with CF. Correlations of free NE activity in sputum supernatant $(a, c, e)$ and surface-bound NE activity on sputum neutrophils $(b, d, f)$ with $a, b)$ neutrophil counts, $c, d)$ levels of interleukin (IL)-8 and e, f) myeloperoxidase (MPO). D/A: donor/acceptor fluorescence. Spearman correlation coefficients $r$ and $p$-values are provided for each correlation.

the pathogenetic relevance of surface-bound NE activity remains unknown [2-4]. In this study, we employed NE-specific FRET reporters designed to localise and quantify NE activity on cell membranes $[21,31]$ and measured surface-bound NE activity on sputum neutrophils from clinically stable adult CF patients with a broad spectrum of severity of lung disease (table 1). We found that surface-bound NE activity was consistently increased on sputum neutrophils from patients with CF compared to healthy 
TABLE 2 Myeloperoxidase (MPO), antiproteases and interleukin (IL)-8 in sputum from controls and cystic fibrosis (CF) patients

\begin{tabular}{lccc} 
& Control & CF & p-value \\
\hline Free $\mathbf{N E}$ activity $\boldsymbol{\mu g} \cdot \mathrm{mL}^{-1}$ & $0.01(0.01-0.02)$ & $23.4(15.2-50.1)$ & $<0.001$ \\
Surface-bound NE activity D/A ratio & $0.09(0.04-0.12)$ & $0.80(0.56-1.07)$ & $<0.001$ \\
$\mathbf{M P O} \boldsymbol{\mu g} \cdot \mathrm{mL}^{-1}$ & $0.4(0.3-0.6)$ & $29.7(18.4-51.8)$ & $<0.001$ \\
$\mathrm{SLPI} \mathbf{n g} \cdot \mathrm{mL}^{-1}$ & $1211.5(923.3-1811.7)$ & $180.8(87.3-474.1)$ & $<0.001$ \\
AAT-NE $\mathbf{n g} \cdot \mathrm{mL}^{-1}$ & $24.8(15.6-33.2)$ & $82.6(40.4-183.1)$ & $<0.001$ \\
IL-8 $\mathbf{n g} \cdot \mathrm{mL}^{-1}$ & $0.6(0.3-0.8)$ & $11.2(8.2-16.6)$ & $<0.001$ \\
\hline
\end{tabular}

Data are presented as median (interquartile range), unless otherwise stated. NE: neutrophil elastase; D/A: donor/acceptor fluorescence; MPO: myeloperoxidase; SLPI: secretory leukocyte protease inhibitor; AATNE: $\alpha_{1}$-antitrypsin-NE complexes.

controls, even in patients with low levels of free NE activity in sputum supernatant (figure 1). Both free and surface-bound NE activity was associated with other markers of airway inflammation, such as sputum neutrophil counts, IL-8, MPO or AAT-NE in our study population (figures 2 and 3) and correlated inversely with FEV1 \% pred in patients with CF (figure 4). In addition, surface-bound NE, but not free NE activity, correlated with FRCpleth \% pred (figure 4). These results suggest that increased membrane-bound
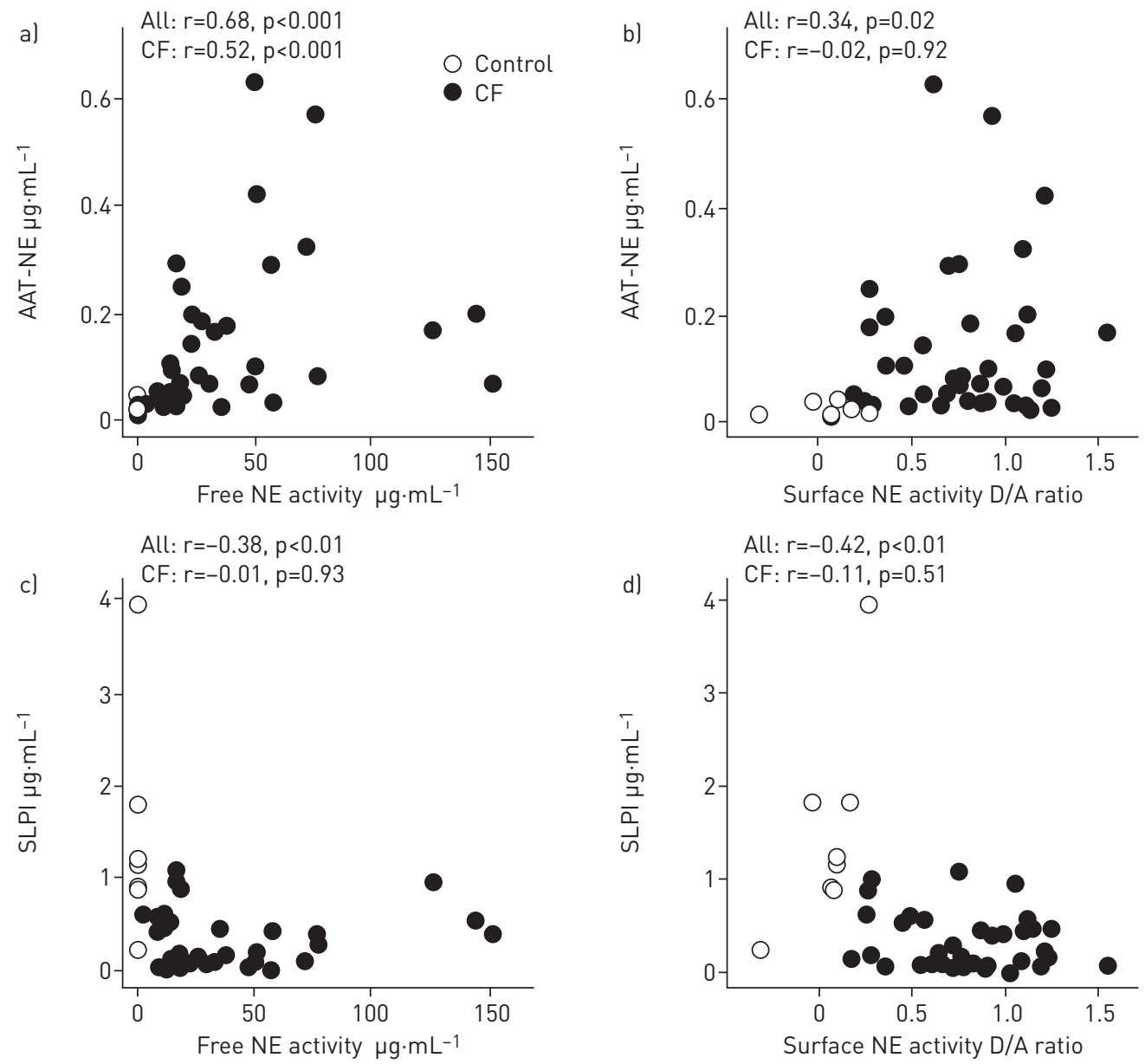

FIGURE 3 Relationship of free and surface-bound neutrophil elastase (NE) activity with antiproteases in sputum from the entire study population lall: control and cystic fibrosis (CFI) and patients with CF. Correlations of $a, c)$ free NE activity in sputum supernatant and b, d) surface-bound NE activity on sputum neutrophils with $a, b) \alpha_{1}$-antitypsin-NE complexes (AAT-NE) and $c, d$ ) secretory leukocyte protease inhibitor (SLPI) in CF sputum. D/A: donor/acceptor fluorescence. Spearman correlation coefficient $r$ and $p$-values are provided for each correlation. 

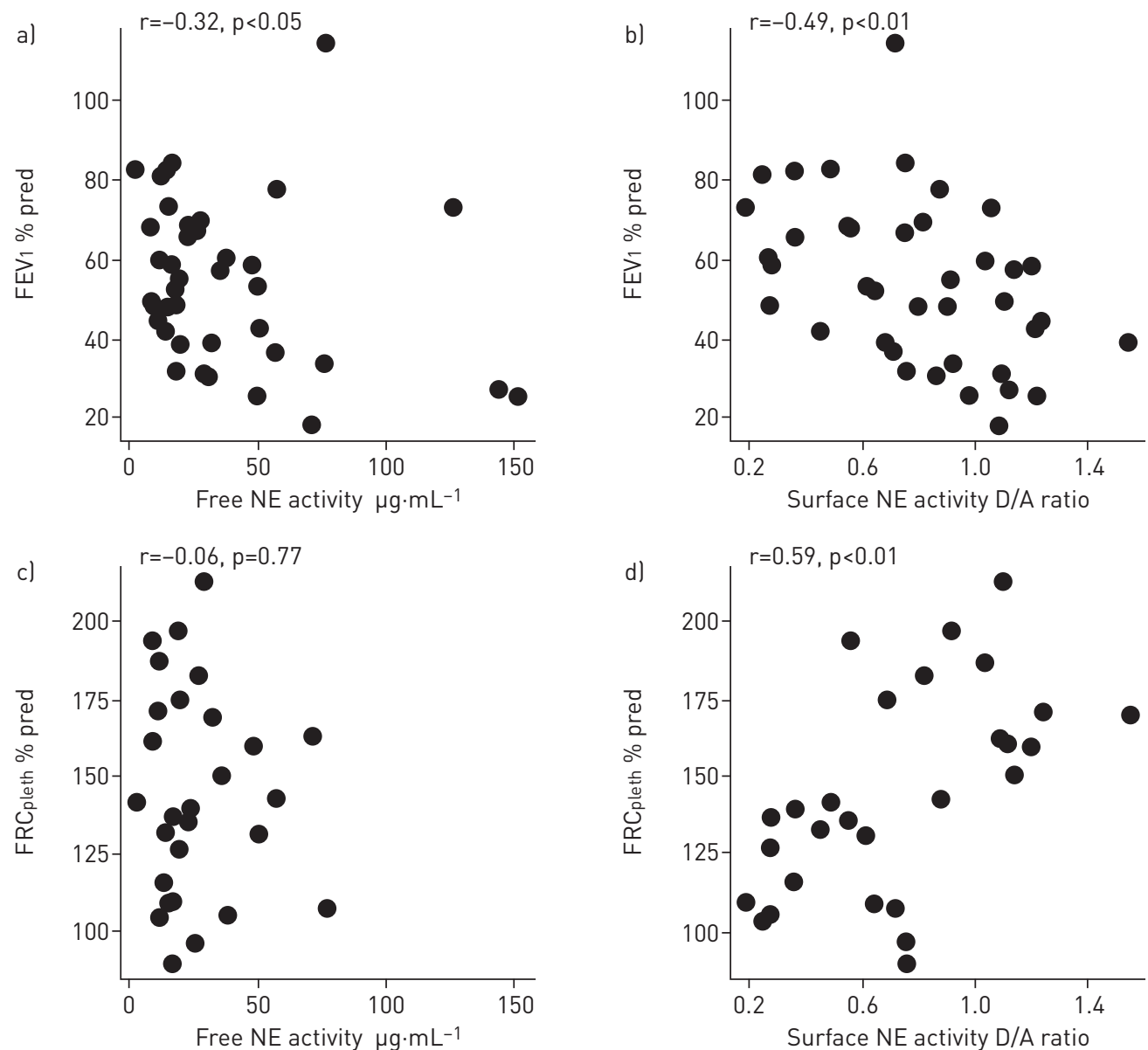

FIGURE 4 Relationship of free and surface-bound neutrophil elastase (NE) activity with lung function parameters of airflow obstruction and air trapping in patients with cystic fibrosis (CF). Correlations of free NE activity in sputum supernatant and surface-bound NE activity on sputum neutrophils with $a, b)$ forced expiratory volume in $1 \mathrm{~s}$ (FEV 1 ) \% predicted and $\mathrm{c}, \mathrm{d}$ ) plethysmographic functional residual capacity (FRCpleth) $\%$ pred. Spearman correlation coefficient $r$ and $p$-values are provided for each correlation.

NE activity on the surface of viable and activated airway neutrophils is associated with airway inflammation, lung function decline and air trapping, and may thus play an important and previously unrecognised role in CF lung disease.

Our study provides novel information on the source of elevated NE activity in CF lung disease. So far, rapid cell death and lysis of neutrophils upon recruitment into CF airways, leading to uncontrolled passive release of NE into the extracellular compartment, has been considered the main mechanism underlying the increased NE burden in CF lung disease [32]. However, a previous study identified a subset of viable neutrophils in CF airways [29, 32]. During homing to the airways, these neutrophils acquired a phenotype of conventional activation and, unexpectedly, showed evidence of mobilisation of NE-rich granula to the cell surface $[29,32]$. Our results obtained with FRET sensors designed to detect membrane-bound NE activity confirm increased expression of NE on the surface of CF sputum neutrophils and demonstrate that this pool of NE has proteolytic activity in CF airways (figure 1). Of note, surface-bound NE activity was consistently increased on CF neutrophils, even when free NE activity in sputum supernatant was low (figure 1). These results suggest that in the microenvironment of CF airways, neutrophils acquire this activated configuration with increased surface-bound $\mathrm{NE}$ activity even at low levels of airway inflammation, when free NE activity is still contained by endogenous antiproteases such as $\alpha_{1}$-antitrypsin and SLPI in the extracellular compartment $[12,27]$.

Of note, surface-bound NE was shown to be largely resistant to inhibition by endogenous inhibitors including $\alpha_{1}$-antitrypsin and SLPI [33]. This suggests that this pool of surface-bound NE activity may be of particular relevance in early lung disease in young children with CF who, probably due to a still-intact antiprotease shield, often show no detectable free NE activity in BAL fluid despite evidence of structural lung disease upon chest CT $[1,2]$. The pathophysiological relevance of increased surface-bound NE 
activity in the context of early $\mathrm{CF}$ lung disease is supported by previous studies in $\beta \mathrm{ENaC}$-overexpressing mice featuring CF-like lung disease with early-onset mucus plugging, airway inflammation and structural lung damage $[22,34,35]$. In this murine model, the level of airway neutrophilia is in the range of $\sim 5-30 \%$ of total cells, thus resembling that of infants and young children with CF and much lower than in adult CF patients, who typically exhibit $\geqslant 90 \%$ neutrophils $[1,2,22,24]$. We showed that the antiprotease shield remains intact and no free $\mathrm{NE}$ activity is detectable in BAL fluid from $\beta E N a C$-overexpressing mice, whereas surface-bound NE activity is consistently increased on airway neutrophils, similar to our findings in $\mathrm{CF}$ patients [22]. Furthermore, we demonstrated that genetic deletion of NE reduced airway inflammation, mucus hypersecretion and structural lung damage in $\beta E N a C$-overexpressing mice $[22,30]$. Collectively, these studies suggest that surface-bound NE activity may be an important player in the pathogenesis of early CF lung disease, and may explain why a subgroup of infants and young children with CF show lung abnormalities by chest CT, even in the absence of free NE activity in BAL fluid $[1,2]$.

Importantly, our data demonstrate that elevated surface-bound NE activity is associated with lung disease severity in adult CF patients who exhibit high levels of neutrophilic airway inflammation and free NE activity. As expected [4, 24, 36], neutrophil counts, IL-8 and MPO as markers of neutrophilic airway inflammation were markedly elevated and free NE activity was readily detectable in sputum samples of our adult patient cohort (figure 2 and table 2). Increased free NE activity was associated with increased levels of NE bound to $\alpha_{1}$-antitrypsin and with reduced levels of SLPI (table 2). Because SLPI is cleaved and inactivated by free NE [12], this pattern is indicative of breakage of the antiprotease shield. In this context of chronic CF airway inflammation, we found that both free and surface-bound NE activity correlated inversely with FEV1 \% pred, the most widely used pulmonary function test to assess airflow limitation and disease progression in CF [2-4] (figure 4). In addition, our data demonstrate that surface-bound NE activity, but not free NE activity, showed a direct correlation with FRCpleth \% pred as a surrogate measure of pulmonary hyperinflation (figure 4). This indicates that surface-bound NE activity may contribute to the severity of CF lung disease independent of free NE in the extracellular compartment. We speculate that this difference may be related to direct effects of surface-bound NE activity that are probably mediated in a focused fashion via direct contact of activated neutrophils with airway epithelial cells, other structural or immune cells, or the extracellular matrix. Pulmonary hyperinflation, as determined from elevated FRCpleth $\%$ pred, may reflect air trapping due to airflow obstruction in the small conducting airways, or hyperinflation due to emphysema related to structural lung damage of distal airspaces. Previous studies in $\beta E N a C$-overexpressing mice demonstrated a role of surface-bound $\mathrm{NE}$ activity in the in vivo pathogenesis of mucus hypersecretion, airway inflammation and emphysema-like structural lung damage [22], and recent chest CT studies detected emphysema formation in adult CF patients [37, 38]. Based on these studies, we speculate that increased surface-bound NE activity on CF airway neutrophils may have disease-promoting effects in small airways and lung parenchyma. Furthermore, our present data suggest that this cell-bound pool of NE activity may be a promising biomarker of disease severity and an important target for therapeutic strategies designed to inhibit NE activity in the lungs of patients with CF.

Our study has several limitations. First, this cross-sectional study does not provide information on the long-term relationship between surface-bound NE activity and lung function decline in patients with CF. Therefore, future longitudinal studies in larger patient cohorts will be required to determine the predictive value of surface-bound NE activity as a biomarker of disease severity and progression. To determine whether surface-bound NE activity predicts pulmonary exacerbations and response to therapy, such studies should include measurements before, at onset and after treatment for pulmonary exacerbation. Second, this study is limited by the lack of chest imaging studies. Future studies including chest CT or magnetic resonance imaging outcome measures $[39,40]$ will be critical to specify and localise the structural abnormalities associated with surface-bound NE that determine lung function decline and pulmonary hyperinflation. Third, established surveillance programmes in infants and young children with CF that include annual BAL will provide opportunities to determine the role of this cell-bound pool of NE in early CF lung disease, where structural lung disease can occur even in the absence of free NE activity in BAL fluid $[1,2]$. We expect that the protocols to capture surface-bound NE activity on airway neutrophils established and employed in the present study will be instrumental for the implementation of these future studies.

In summary, this study demonstrates for the first time that NE activity on sputum neutrophils is associated with severity of lung disease in patients with CF. We found that NE activity is consistently increased on the surface of CF sputum neutrophils, even at low levels of free NE activity in sputum supernatant. Furthermore, we demonstrate that surface-bound NE activity correlates with pulmonary function indices of airflow limitation and pulmonary hyperinflation. These results suggest that surface-bound NE activity, probably reflecting a freshly secreted pool of NE that is not inhibited by endogenous antiproteases, may play an important role in the pathogenesis and serve as a novel biomarker of disease severity in patients with CF and potentially other chronic neutrophilic lung diseases. 


\section{Acknowledgements}

The authors thank all the patients with cystic fibrosis for their participation in this study; Yvonne Koch and Susanne Korsten (Thoraxklinik at the University Hospital Heidelberg, Heidelberg, Germany) for assistance in sample collection; and Nicole Heath, Frank Stein, Aliaksandr Halavatyi and Marko Lampe (TLRC Microscopy Laboratory, Heidelberg) for expert assistance in microscopy and data analysis.

\section{References}

1 Sly PD, Brennan S, Gangell C, et al. Lung disease at diagnosis in infants with cystic fibrosis detected by newborn screening. Am J Respir Crit Care Med 2009; 180: 146-152.

2 Sly PD, Gangell CL, Chen L, et al. Risk factors for bronchiectasis in children with cystic fibrosis. $N$ Engl J Med 2013; 368: 1963-1970.

3 Sagel SD, Wagner BD, Anthony MM, et al. Sputum biomarkers of inflammation and lung function decline in children with cystic fibrosis. Am J Respir Crit Care Med 2012; 186: 857-865.

4 Mayer-Hamblett N, Aitken ML, Accurso FJ, et al. Association between pulmonary function and sputum biomarkers in cystic fibrosis. Am J Respir Crit Care Med 2007; 175: 822-828.

5 Chalmers JD, Moffitt KL, Suarez-Cuartin G, et al. Neutrophil elastase activity is associated with exacerbations and lung function decline in bronchiectasis. Am J Respir Crit Care Med 2017; 195: 1384-1393.

6 Voynow JA, Fischer BM, Malarkey DE, et al. Neutrophil elastase induces mucus cell metaplasia in mouse lung. Am J Physiol Lung Cell Mol Physiol 2004; 287: L1293-L1302.

$7 \quad$ Fahy JV, Dickey BF. Airway mucus function and dysfunction. N Engl J Med 2010; 363: 2233-2247.

8 Owen CA. Roles for proteinases in the pathogenesis of chronic obstructive pulmonary disease. Int $J$ Chron Obstruct Pulmon Dis 2008; 3: 253-268.

9 Pham CT. Neutrophil serine proteases: specific regulators of inflammation. Nat Rev Immunol 2006; 6: 541-550.

10 Lee WL, Downey GP. Leukocyte elastase: physiological functions and role in acute lung injury. Am J Respir Crit Care Med 2001; 164: 896-904.

11 Taggart CC, Greene CM, Carroll TP, et al. Elastolytic proteases: inflammation resolution and dysregulation in chronic infective lung disease. Am J Respir Crit Care Med 2005; 171: 1070-1076.

12 Weldon S, McNally P, McElvaney NG, et al. Decreased levels of secretory leucoprotease inhibitor in the Pseudomonas-infected cystic fibrosis lung are due to neutrophil elastase degradation. J Immunol 2009; 183: 8148-8156.

13 Hirche TO, Benabid R, Deslee G, et al. Neutrophil elastase mediates innate host protection against Pseudomonas aeruginosa. J Immunol 2008; 181: 4945-4954.

14 Hartl D, Latzin P, Hordijk P, et al. Cleavage of CXCR1 on neutrophils disables bacterial killing in cystic fibrosis lung disease. Nat Med 2007; 13: 1423-1430.

15 Le Gars M, Descamps D, Roussel D, et al. Neutrophil elastase degrades cystic fibrosis transmembrane conductance regulator via calpains and disables channel function in vitro and in vivo. Am J Respir Crit Care Med 2013; 187: 170-179.

16 Caldwell RA, Boucher RC, Stutts MJ. Neutrophil elastase activates near-silent epithelial $\mathrm{Na}^{+}$channels and increases airway epithelial $\mathrm{Na}^{+}$transport. Am J Physiol Lung Cell Mol Physiol 2005; 288: L813-L819.

17 Mall MA, Hartl D. CFTR: cystic fibrosis and beyond. Eur Respir J 2014; 44: 1042-1054.

18 Ratjen $\mathrm{F}$, Waters $\mathrm{V}$, Klingel $\mathrm{M}$, et al. Changes in airway inflammation during pulmonary exacerbations in patients with cystic fibrosis and primary ciliary dyskinesia. Eur Respir J 2016; 47: 829-836.

19 Owen CA, Campbell MA, Boukedes SS, et al. Cytokines regulate membrane-bound leukocyte elastase on neutrophils: a novel mechanism for effector activity. Am J Physiol 1997; 272: L385-L393.

20 Campbell EJ, Owen CA. The sulfate groups of chondroitin sulfate- and heparan sulfate-containing proteoglycans in neutrophil plasma membranes are novel binding sites for human leukocyte elastase and cathepsin G. J Biol Chem 2007; 282: 14645-14654.

21 Gehrig S, Mall MA, Schultz C. Spatially resolved monitoring of neutrophil elastase activity with ratiometric fluorescent reporters. Angew Chem Int Ed 2012; 51: 6258-6261.

22 Gehrig S, Duerr J, Weitnauer M, et al. Lack of neutrophil elastase reduces inflammation, mucus hypersecretion, and emphysema, but not mucus obstruction, in mice with cystic fibrosis-like lung disease. Am J Respir Crit Care Med 2014; 189: 1082-1092.

23 De Boeck K, Derichs N, Fajac I, et al. New clinical diagnostic procedures for cystic fibrosis in Europe. J Cyst Fibros 2011; 10: Suppl. 2, S53-S66.

24 Hector A, Jonas F, Kappler M, et al. Novel method to process cystic fibrosis sputum for determination of oxidative state. Respiration 2010; 80: 393-400.

25 Miller MR, Hankinson J, Brusasco V, et al. Standardisation of spirometry. Eur Respir J 2005; 26: 319-338.

26 Wanger J, Clausen JL, Coates A, et al. Standardisation of the measurement of lung volumes. Eur Respir J 2005; 26 : 511-522.

27 Greene CM, McElvaney NG. Proteases and antiproteases in chronic neutrophilic lung disease - relevance to drug discovery. Br J Pharmacol 2009; 158: 1048-1058.

28 McGarvey LPA, Dunbar K, Martin SL, et al. Cytokine concentrations and neutrophil elastase activity in bronchoalveolar lavage and induced sputum from patients with cystic fibrosis, mild asthma and healthy volunteers. J Cyst Fibros 2002; 1: 269-275.

29 Tirouvanziam R, Gernez Y, Conrad CK, et al. Profound functional and signaling changes in viable inflammatory neutrophils homing to cystic fibrosis airways. Proc Natl Acad Sci USA 2008; 105: 4335-4339.

30 Wagner CJ, Schultz C, Mall MA. Neutrophil elastase and matrix metalloproteinase 12 in cystic fibrosis lung disease. Mol Cell Pediatr 2016; 3: 25.

31 Hu H-Y, Gehrig S, Reither G, et al. FRET-based and other fluorescent proteinase probes. Biotechnol J 2014; 9: 266-281.

32 Margaroli C, Tirouvanziam R. Neutrophil plasticity enables the development of pathological microenvironments: implications for cystic fibrosis airway disease. Mol Cell Pediatr 2016; 3: 38. 
33 Owen CA, Campbell MA, Sannes PL, et al. Cell surface-bound elastase and cathepsin G on human neutrophils: a novel, non-oxidative mechanism by which neutrophils focus and preserve catalytic activity of serine proteinases. J Cell Biol 1995; 131: 775-789.

34 Mall M, Grubb BR, Harkema JR, et al. Increased airway epithelial $\mathrm{Na}^{+}$absorption produces cystic fibrosis-like lung disease in mice. Nat Med 2004; 10: 487-493.

35 Zhou Z, Duerr J, Johannesson B, et al. The ENaC-overexpressing mouse as a model of cystic fibrosis lung disease. J Cyst Fibros 2011; 10: Suppl. 2, S172-S182.

36 Sloane AJ, Lindner RA, Prasad SS, et al. Proteomic analysis of sputum from adults and children with cystic fibrosis and from control subjects. Am J Respir Crit Care Med 2005; 172: 1416-1426.

37 Wielpütz MO, Weinheimer $\mathrm{O}$, Eichinger $\mathrm{M}$, et al. Pulmonary emphysema in cystic fibrosis detected by densitometry on chest multidetector computed tomography. PLoS One 2013; 8: e73142.

38 Mets OM, Roothaan SM, Bronsveld I, et al. Emphysema is common in lungs of cystic fibrosis lung transplantation patients: a histopathological and computed tomography study. PLoS One 2015; 10: e0128062.

39 Szczesniak R, Turkovic L, Andrinopoulou E-R, et al. Chest imaging in cystic fibrosis studies: what counts, and can be counted? J Cyst Fibros 2017; 16: 175-185.

40 Wielpütz M, Eichinger M, Biederer J, et al. Imaging of cystic fibrosis lung disease and clinical interpretation. Rofo 2016; 188: 834-845. 